\title{
Plasma CA 19-9 in Advanced Non-Small Cell Lung Cancer
}

\author{
Ahmed Abdallah ${ }^{1}$, Mohamed Wahba ${ }^{1}$, Ahmed El Bastawisy ${ }^{2}, \&$ Rabab Gaafar ${ }^{2}$ \\ ${ }^{1}$ Faculty of Pharmacy, Al-Azhar University, Cairo, Egypt \\ ${ }^{2}$ National Cancer Institute, Cairo University, Medical Oncology, Cairo, Egypt \\ Correspondence: Ahmed El Bastawisy, National Cancer Institute, Kasr El-Aini Street, Fom El-khalig Square, \\ Cairo, Egypt. Tel: 20-100-110-4774. Fax: 20-022-270-7988. E-mail: a_s_basta@ hotmail.com
}

Received: May 31, 2013 Accepted: June 17, 2013 Online Published: July 22, 2013

doi:10.5539/cco.v2n2p11 URL: http://dx.doi.org/10.5539/cco.v2n2p11

\begin{abstract}
Background: Lung cancer is the leading cause of cancer death worldwide however no serum marker is routinely recommended till now.

Patients and Methods: This is a prospective case control study including two groups of adults, Group I: Includes healthy volunteers as controls. Group II: Includes patients with advanced non-small cell lung cancer (NSCLC). Plasma CA19-9 levels were measured at baseline by ELISA before first line chemotherapy. Primary end point was comparison between the levels of CA19-9 in patients and controls. Secondary endpoint was correlation between CA19-9 level and clinical response (CR), progression free survival (PFS) and overall survival (OS) in advanced NSCLC.

Results: 34 healthy adults and 35 patients with advanced NSCLC were included and followed up during the period from October 2009 to February 2013 with median follow up of 10.5 months. The mean and median pre-treatment plasma CA 19-9 concentrations of NSCLC patients were 240.7 and $231 \mathrm{U} / \mathrm{ml}$ respectively. There was statistically significant difference between the patient and control groups ( $\mathrm{p}<0.001)$. No significant correlations were found between CA19-9 levels and CR, PFS and OS p-values: 0.5, 0.3 and 0.6 respectively Conclusion: Plasma CA19-9 is a reliable marker for advanced NSCLC.
\end{abstract}

Keywords: CA19-9 Non-small cell lung cancer

\section{Introducation}

Among malignant tumors lung cancer is the leading cause of death worldwide (Jemal, 2008). Unfortunately, its prognosis is very poor; the disease is curable rarely with an estimated 5-year overall survival rate of 15\% (Sun, 2007), therefore the development of novel diagnostic techniques to identify the disease in the early stages and to follow up its progression is important for more effective treatment and improved prognosis.

Carbohydrate antigen 19-9 (CA 19-9) was originally isolated from a cell line of a human colorectal cancer as a mucin like product (Koprowski, 1979). The antigen is found in the several tissues, as the epithelial cells of the gall bladder, biliary ducts, pancreas and stomach (Magnai, 1983). CA 19-9 antigen in tissue exists primarily as an epitope present on a glycolipid, sialolacto- N fucopentose II ganglioside; in serum, the CA 199 antigen is associated with a mucin (Magnani, 1982). CA 19-9 was found to be elevated in both benign and malignant conditions, although the level in malignancy is significantly higher. CA 19-9 was found to be elevated in many types of malignancies as the pancreatic, colorectal, hepatic, lung, and ovarian cancers. Benign conditions associated with high levels of CA 19-9 including hepatobiliary system disease, pleural effusion, pneumonia, SLE, and renal failure (Pavai, 2003). The CA 19-9 is not a tumor specific, but it is a tumor associated antigen. CA 19-9 is synthesized normally by human pancreatic and biliary ductular cells, gastric, colonic, endometrial and salivary epithelia (Rhodes, 1990) and has been detected in the normal seminal fluid. This explains the high levels of CA 19-9 in many malignancies.

Since its discovery by Koprowski and coworkers, CA 199 antigen has been used widely as a tool for the investigation and management of patients with pancreatic carcinoma.

CA 19-9 has been studied in relation to gastrointestinal cancer and has been shown to have a good clinical value as a prognostic and monitoring tool for the follow up of therapy effectiveness of (Koprowski, 1981; Gupta, 1985). Multiple studies have shown that while high CA 19-9 serum levels appear to be useful in the diagnosis of 
adenocarcinoma of the upper gastrointestinal tract and in monitoring of colon cancer, its greatest sensitivity is in the detection of pancreatic adenocarcinoma (Safi, 1986). Elevations in CA 19-9 level correlate with the degree of tumor differentiation and the extent of tumor mass (Malesci, 1987).

Pavai Sthaneshwar and S. F. Yap have observed marked elevation of CA 19-9 in carcinoma of lung with metastasis. CA 19-9 had been found in epithelial tumors of the lung (Ohshio, 1995) explaining the presence of elevated levels of this antigen in lung cancer. High levels CA 19-9 were reported to be related to advanced stage adenocarcinoma of the lung (Mizushima, 1991).

Niklinski J, and his colleagues, proved that preoperative CEA, CA 19-9 and SCC-Ag have shown a prognostic value in patients with NSCLC. Patients with high preoperative levels had shown worse prognosis than patients who had lower levels (Niklinski, 1992).

\subsection{Aim of Work}

1) Comparison between the levels of CA19-9 in patients with advanced NSCLC and controls.

2) Assessment of correlation between CA19-9 level and clinical outcome including clinical response (CR), progression free survival (PFS) and overall survival (OS).

\section{Patients and Methods}

This study was conducted on 69 adults, aged 39-77 years old who were classified into 2 groups;

\subsection{Control Group}

This group constitutes the healthy volunteers who are not complaining of any chronic or acute diseases including any respiratory problems, and who are not on regular medications. They have been collected randomly without any inclusion or exclusion criteria except being healthy as described earlier. They are 34.

\subsection{Patient Group}

This group constitutes the Non-Small Cell Lung Cancer (NSCLC) patients. Samples were collected from patients presented to chest section of the medical oncology department, National Cancer Institute (NCI), Cairo University, randomly with no selections except for the criteria of having a confirmed diagnosis of NSCLC, and an advanced stage of it (Stage III or IV). Those are 35 patients.

All patients were newly diagnosed, and did not receive chemotherapy, radiotherapy or subjected to surgical resection of the cancer.

This study was conducted according to Helsinki's declaration and the guidelines for Good Clinical Practice. The local ethics committees approved the protocol, and informed consent was obtained from all patients before study entry.

\subsubsection{Inclusion Criteria}

Patient must have a histologically confirmed diagnosis of lung cancer. Stage IIIB \& Stage IV lung cancer. Controls are healthy volunteers with no known acute or chronic illness. Patients are at least 18 years of age. Patients have a performance status of (ECOG Scale) $\leq 2$, with a life expectancy of at least 6 months. Patient has adequate bone marrow function, (WBC count $\geq 3.0 \times 109 / \mathrm{L}, \mathrm{ANC} \geq 1.5 \times 109 / \mathrm{L}$ platelet count $\geq 100 \times 109 / \mathrm{L}$, hemoglobin level $\geq 9 \mathrm{~g} / \mathrm{L}$. Patients also have an adequate liver and kidney functions. Patients should have compliance, mental state and geographic proximity that allow adequate follow up and they have to provide written informed consent before any study-specific procedure.

\subsubsection{Exclusion Criteria}

Patients who are pregnant, breastfeeding, patients with a currently active second malignancy, or patients who are currently involved in another clinical trial.

\subsection{Treatment Plan}

Patients with NSCLC received the following chemotherapy regimen:

Gemcitabine: $1000 \mathrm{mg} / \mathrm{m}^{2}$, IV on $250 \mathrm{cc}$ NS over $30 \mathrm{~min}, \mathrm{D} 1,8$.

Cisplatin: $80 \mathrm{mg} / \mathrm{m}^{2} \mathrm{D}$, IV on $500 \mathrm{cc}$ NS over $1 \mathrm{hr}$. with standard hydration, D1.

Every 3 weeks, up to 6 cycles in responding patients. Evaluation was done every 6 weeks.

\subsection{Study Assessment}

Pretreatment assessment included complete medical history and physical examination. 
Addition assessment was done within 7 days before treatment included vital signs, performance status (ECOG), Complete blood count (CBC) with differential and full biochemical panel, liver and renal function and repeated before each treatment course.

Radiological evaluation including computerized tomography (CT) scan of the chest and U/S Abdomen \& pelvis were done. Additional radiological imaging such as bone scan was done if indicated.

Evaluation was done according to RECIST as follows: A complete response (CR) is defined as complete disappearance of all known disease determined by two observations not less than 4 weeks apart. A partial response (PR) means $30 \%$ or greater reduction of the product of the perpendicular diameters of all measurable lesions. Stable disease (SD) defined as less than $30 \%$ reduction or less than $20 \%$ increase in tumor size. Progressive disease (PD) was an increase of more than $20 \%$ in the product of the perpendicular diameters of all measurable lesions or the appearance of new lesions.

\subsection{Post Treatment Evaluation}

Medical history and physical examination were taken every 3 weeks, $\mathrm{CBC}$ and chemistry tests were done every 3 weeks, while CT chest and upper abdomen were done every 6 weeks. Other investigations were done if indicated.

\subsection{Statistical Methods}

Data management and analysis were performed using Statistical Package for Social Sciences (SPSS) vs. 17.

Numerical data were summarized using means and standard deviations or median \& ranges. Comparisons between the 2 groups were performed using the Student's t-test (Dawson \& Trapp, 2001).

All p-values are two-sided. P-values $<0.05$ were considered significant.

\subsection{Samples Collection and Storage}

$5 \mathrm{~mL}$ of venous blood samples were withdrawn by EDTA.K2 containing BD vacutainers - those with the purple cap- from patients and control. We centrifuged the samples at 4000 r.p.m for 15 minutes, and plasmas were separated and stored at $<-20^{\circ} \mathrm{C}$ till the time of analysis. We used ELISA to assess CA 19-9 plasma levels.

\subsection{Test Principle}

The essential reagents required for an immunoenzymometric assay include high affinity and specificity antibodies (enzyme and immobilized), with different and distinct epitope recognition, in excess, and native antigen. In this procedure, the immobilization takes place during the assay at the surface of a microplate well through the interaction of streptavidin coated on the well and exogenously added biotinylated monoclonal anti-CA19-9 antibody.

Upon mixing monoclonal biotinylated antibody, and a serum containing the native antigen, reaction results between the native antigen and the antibody, forming an antibody-antigen complex.

Simultaneously, the complex is deposited to the well through the high affinity reaction of streptavidin and biotinylated antibody. This interaction is illustrated below:

$$
\begin{gathered}
\mathrm{Ag}(\mathrm{CA} 19-9)+\mathrm{Btn} \mathrm{Ab}(\mathrm{m})+\text { Streptavidin } \mathrm{CW} \rightarrow \text { Immobilized complex (IC) } \\
\text { Streptavidin CW }=\text { Streptavidin immobilized on well } \\
\text { Immobilized complex }(\mathrm{IC})=\mathrm{Ag}-\mathrm{Ab} \text { bound to the well }
\end{gathered}
$$

After a suitable incubation period, the antibody-antigen bound fraction is separated from unbound antigen by decantation or aspiration. Another antibody (directed at a different epitope) labeled with an enzyme is added. Another interaction occurs to form an enzyme labeled antibody-antigen-biotinylated-antibody complex on the surface of the wells. Excess enzyme is washed off via a wash step. A suitable substrate is added to produce color measurable with the use of a microplate spectrophotometer. The enzyme activity on the well is directly proportional to the native free antigen concentration. By utilizing several different serum references of known antigen concentration, a dose response curve can be generated from which the antigen concentration of an unknown can be ascertained.

\subsection{Test Procedures}

Reagents, samples and controls, all were brought to room temperature $\left(20-27^{\circ} \mathrm{C}\right) .0 .025 \mathrm{ml}(25 \mu \mathrm{l})$ of the serum reference, control or specimen were pipetted into the assigned well, then we added $0.100 \mathrm{ml}(100 \mu \mathrm{l})$ of the biotinylated labeled antibody to every well, then we swirled the microplates gently for 20-30 seconds and covered it. The microplates were incubated for 60 minutes at room temperature. Then, we discarded the contents of each microplate by aspiration, and added $300 \mu 1$ of wash buffer, aspirated and repeated the step of the addition 
of $0.100 \mathrm{ml}(100 \mu \mathrm{l})$ of the biotinylated labeled antibody to every well, etc for a total of three (3) washes. 0.100 $\mathrm{ml}(100 \mu \mathrm{l})$ of the Ca19-9 Enzyme Reagent labeled antibody was added to each well, then we covered and incubated for 60 minutes at room temperature. The contents of each microplate were discarded by aspiration. 300 $\mu \mathrm{l}$ of wash buffer was added, we aspirated and repeated the step of the addition of $0.100 \mathrm{ml}(100 \mu \mathrm{l})$ of the biotinylated labeled antibody to every well, etc again for a total of 3 washes. $0.100 \mathrm{ml}(100 \mu \mathrm{l})$ of working substrate solution was added to all wells, and then we incubated for 15 minutes at room temperature. Finally we added $0.050 \mathrm{ml}$ of stop solution each well and mixed gently.

The absorbance was red in each well at $450 \mathrm{~nm}$ (using a reference wave length of 620-630 $\mathrm{nm}$ to minimize well imperfections) in a reader of microplate.

\section{Results}

This study was carried out on 35 patients with NSCLC presented to medical oncology department, National Cancer Institute, Cairo University during the period from October 2009 till February 2013 with a median follow up period of 10.5 months. In addition 34 age and sex matched normal subjects were used as control group. There were 28 males $(80 \%)$ and 7 females $(20 \%)$, their ages ranged from 39 to 77 years. Patients' characteristics are summarized in Table 1.

Table 1. Clinicopathological characteristics of the 35 NSCLC patients included in the study

\begin{tabular}{ll}
\hline Characteristics & Number $(\%)$ \\
\hline Number & $35(100)$ \\
Sex & \\
$\quad$ Female & $7(20)$ \\
$\quad$ Male & $28(80)$ \\
Age (Years) & \\
$\quad$ Range & $39-77$ \\
$\quad$ Median & 58 \\
Pathological subtype & \\
$\quad$ Adenocarcinoma & $18(51.4)$ \\
$\quad$ Squamous Cell Carcinoma & $12(34.3)$ \\
Large Cell Carcinoma & $5(14.3)$ \\
Stage & $12(34)$ \\
III & $23(66)$ \\
IV & \\
Smoking & $29(83)$ \\
Smoker & $6(17)$ \\
$\quad$ Non-Smoker &
\end{tabular}

\subsection{CA 19-9 Plasma Levels}

The pre-treatment plasma CA 19-9 concentrations of our NSCLC patients ranged from 155 to $851 \mathrm{U} / \mathrm{ml}$, the mean and median were 240.7 and $231 \mathrm{U} / \mathrm{ml}$ respectively. There was statistically significant difference between the patient and control groups $(\mathrm{p}<0.001)$ as illustrated in Table 2.

Table 2. Comparison of the plasma levels of CA 19-9 in NSCLC patients and controls groups

\begin{tabular}{lcc}
\hline Points of comparisons & \multicolumn{2}{c}{ Group } \\
\cline { 2 - 3 } & Cases $\mathrm{n}=35$ & Control $\mathrm{n}=34$ \\
\hline Mean & 240.7 & 4.4 \\
$\mathrm{SD}(\sigma)$ & 47.7 & 4.9 \\
Minimum & 155 & 1 \\
Median & 231 & 4.5 \\
Maximum & 349 & 15 \\
P-Value & & $<0.001$ \\
\hline
\end{tabular}


Figure 1 shows the comparison between the mean plasma levels of CA 19-9 in the patient and control groups.

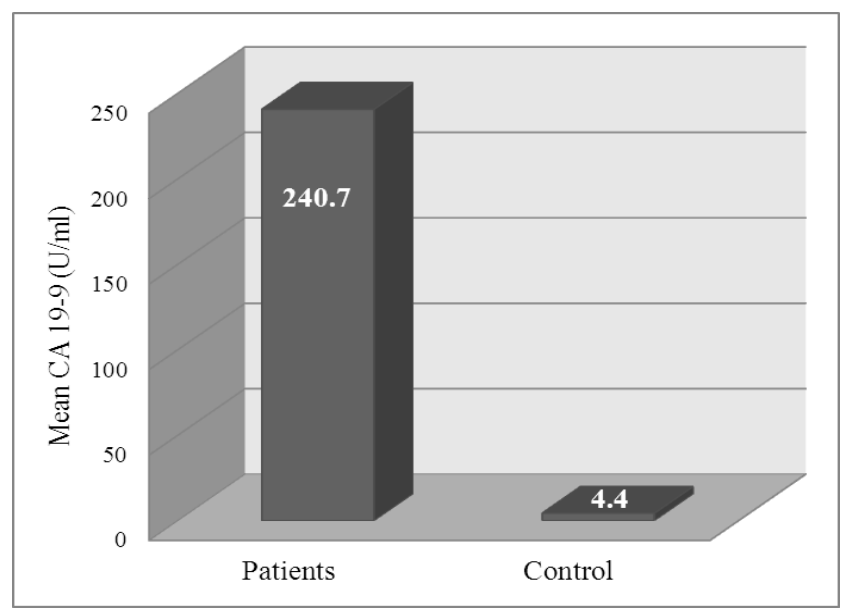

Figure 1. Comparison of mean plasma levels of CA 19-9 in NSCLC patients and controls groups

\subsection{Correlation of plasma CA 19-9 with clinico-pathologic parameters}

\section{Correlation of plasma CA 19-9 level with age and gender:}

There were no significant associations between CA 19-9 levels and age

$(\mathrm{P}$ value $=0.06)$ or gender $(\mathrm{P}$ value $=0.63)$.

\section{Correlation of plasma CA 19-9 level with Pathological subtypes:}

Our patients with adenocarcinoma had a mean plasma CA 19-9 level of 240.2 \pm 46.5 SD compared to a mean level of $247.7 \pm 54.8 \mathrm{SD}$ for patients with other pathological subtypes. However, this correlation wasn't statistically significant $(\mathrm{p}=0.64)$.

\section{Correlation of plasma CA 19-9 level with Stage:}

Patients were categorized as stage III and stage IV. Stage III patients had mean plasma CA 19-9 5 level of $233.77 \pm$ 53.87 SD and patients categorized as stage IV had a mean plasma CA 19-9 level of 239.9 $\pm 48.47 \mathrm{SD}$. However, this difference did not reach statistical significance $(\mathrm{p}=0.7)$.

\section{Correlation of plasma CA 19-9 level with clinical response of the patients:}

Patients who had achieved CR, PR, and SD are 17, and Surprisingly 8 of them had low levels of CA 19-9 ( $\leq 243$ $\mathrm{U} / \mathrm{ml}$ ) and 9 had high levels (>243 $\mathrm{U} / \mathrm{ml}),(\mathrm{p}=0.5)$.

The patients group who had PD was 18. 9 had low levels and 9 had high levels ( $\mathrm{p}=0.9)$, as illustrated in Table 3.

Table 3. Correlation of plasma CA 19-9 level with clinical response

\begin{tabular}{cccc}
\hline \multicolumn{2}{c}{ CR,CR, PR, SD } & & PD \\
\hline High & Low & High & Low \\
9 & 8 & 9 & 9 \\
P-value & 0.5 & P-value & 0.9 \\
\hline
\end{tabular}

CR: Complete remission $\quad$ PR: Partial remission $\quad$ SD: Stationary disease $\quad$ PD: Progressive disease

\section{Correlation of plasma CA 19-9 level with Progression Free Survival and Overall Survival of the patients:}

The median plasma CA 19-9 level of our NSCLC patients were $231 \mathrm{U} / \mathrm{ml}$, ranging from 155 to $851 \mathrm{U} / \mathrm{ml}$ (mean 240.7 U/ml $\pm 47.7 \mathrm{U} / \mathrm{ml} \mathrm{SD})$. Patients were divided into high CA 19-9 (>243 U/ml) or low CA 19-9 ( $\leq 243 \mathrm{U} / \mathrm{ml})$ group using the median value as a cut-off.

The difference in the median progression free survival of the patients with high serum CA 19-9 level was not 
statistically significant (3 months) compared with those with low CA 19-9 level (5 months). P value $=0.3$.

There was no statistically significance difference also in overall survival as illustrated in Table 4. Figure 2 shows the correlation of plasma CA 19-9 level with progression free survival and overall survival of the patients

Table 4. Correlation of plasma CA 19-9 level with progression free survival and overall survival of the patients

\begin{tabular}{llll}
\hline CA 19-9 level & Total No. of patients & Median PFS & Median OS \\
\hline Low $(\leq 243 \mathrm{U} / \mathrm{ml})$ & 17 & 5 & 9 \\
High $(>243 \mathrm{U} / \mathrm{ml})$ & 18 & 3 & 8 \\
P-value & & 0.3 & 0.6 \\
\hline
\end{tabular}

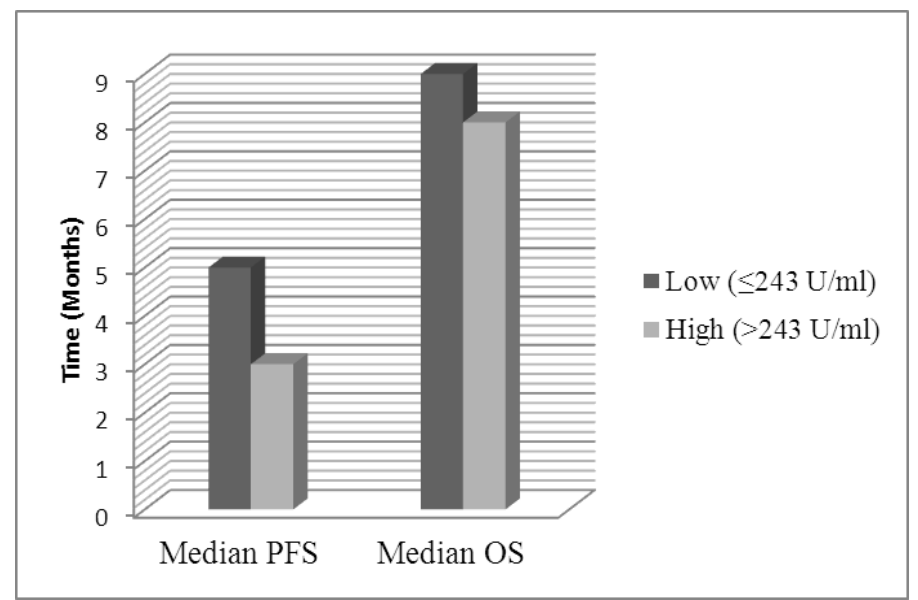

Figure 2. Correlation of plasma CA 19-9 level with Progression Free Survival and Overall Survival of the patients

\section{Discussion}

Worldwide, cancer is the $2^{\text {nd }}$ leading cause of death - following heart diseases, with lung cancer being the leading cause of cancer deaths in men and the $2^{\text {nd }}$ cause of cancer deaths in women, with an estimated 951,000 deaths in men and 427,400 deaths in women in 2008.

Unfortunately, the disease prognosis is poor, and it is rarely curable with an estimated 5-year overall survival rate of $15 \%$ (Sun, 2007). The cure rates of lung cancer have been relatively unaltered during the past 40 years. The high mortality rate is attributed to the low cure rate $(6-15 \%)$ which in turn is attributed to the lack of adequate screening and early detection measures, and therefore, new strategies for screening and treatment of this disease are necessary for the improvement of patients' outcome (Auberger, 2006).

\subsection{CA 19-9 Plasma Levels}

Statistically significant difference was obtained on comparing CA19-9 plasmas levels in patient group with the control group. CA19-9 concentration means were 240.66 and $4.4 \mathrm{U} / \mathrm{ml}$, and ranges of (155-349 $\mathrm{U} / \mathrm{ml})$ and (1-15 $\mathrm{U} / \mathrm{ml}$ ), for the patients and control groups respectively.

CA19-9 plasmas levels were high in all of our patients. These results are the same as reported by Indranath and his colleagues, who have found CA19-9 to be high in their patient group compared to the control group (Ghosh, 2013).

Indranath and his colleagues have reported that 'among the cases, values for different tumor markers- CEA, CA15-3, CA19-9 and CA125- in BAL fluid were similar among smokers and non smokers except that of CA19-9 which was high only in smokers' and all our patients were smokers or passive smokers. Niklinski J, and his colleagues have found CA19-9 to be elevated only in $44.2 \%$ of their patients (Niklinski, 1992).

\subsection{CA 19-9 Clinicopathological Correlations}

In our study CA 19-9 didn't show any correlation with age $(\mathrm{P}=0.06)$, sex $(\mathrm{P}=0.63)$, histopathological subtype $(\mathrm{P}=0.64)$, or stage $(\mathrm{P}=0.7)$.

Buccheri, et al, 1987, didn't also find any correlation between CA 19-9, and histological type of the lung cancer 
(Buccheri, 1987). On the other hand Niklinski J, and his colleagues observed CA 19-9 elevation to be most frequently with adenocarcinoma, more than the other histopathological subtypes (Niklinski, 1992).

Niklinski J, and his colleagues has also reported that elevated CA 19-9 levels correlate with the pathological stage only in adenocarcinoma, some results which didn't go in agreement with ours.

\subsection{Correlation of plasma CA 19-9 Level with Progression Free Survival and Overall Survival}

Patients in the present study were divided into high CA 19-9 (>243 U/ml) or low CA 19-9 ( $\leq 243 \mathrm{U} / \mathrm{ml})$ plasma levels groups using the median value as a cut-off. The median CA 19-9 plasma level of our NSCLC patients was $231 \mathrm{U} / \mathrm{ml}$, ranging from 155 to $851 \mathrm{U} / \mathrm{ml}$ (mean $240.7 \mathrm{U} / \mathrm{ml} \pm 47.7 \mathrm{U} / \mathrm{ml} \mathrm{SD}$ ).

There was no statistically significant difference in the median progression free survival of the patients with high serum CA 19-9 level (3 months) compared with those with low CA 19-9 level (5 months). $\mathrm{P}$ value $=0.3$.

There was no statistical significance has been noted in both groups, the low or the high CA 19-9 groups with the OS. OS range was (3-22 months) with a median of 8.5. We strongly believe that the lack of correlation between the clinical parameters, overall survival, and progression free survival with CA 19-9 plasma levels mainly is due to the fact that the low $(\leq 243 \mathrm{U} / \mathrm{ml})$ and high $(>243 \mathrm{U} / \mathrm{ml})$ CA 19-9 levels are actually high levels, taking into consideration that the normal range of CA $19-9$ in healthy individual is $0-37 \mathrm{U} / \mathrm{ml}$, and that the lowest value in our patient was $155 \mathrm{U} / \mathrm{ml}$. Also the relatively low patients' number could be a contributing factor for that missing link between the CA 19-9 plasma levels and the clinical response.

\section{Conclusions}

1) CA 19-9 plasma levels were found to be higher in patient group in comparison to the control group.

2) CA 19-9 plasma levels in the advanced stages - III and IV - of NSCLC don't correlate with age, gender, stage, or histopathological subtype.

3) High and low plasma levels of CA 19-9 in advanced stages - III and IV - of NSCLC don't correlate with the patients' overall survival or progression free survival.

4) CA 19-9 level was found to be much higher in our study in the patient group compared to the control group, but more studies are still needed both to validate this finding and for assessing its levels before and after treatment trying to elucidate its value in monitoring the disease, rather than its role as a diagnostic tool.

\section{References}

Jemal, A., Siegel, R., Ward, E., Hao, Y., Xu, J., Murray, T., \& Thun, M. J. (2008). Cancer statistics 2008. CA Cancer J Clin, 58(2), 71-96. http://dx.doi.org/10.3322/CA.2007.0010

Sun, S., Schiller, J. H., Spinola, M., \& Minnal, J. D. (2007). New molecularly targeted therapies for lung cancer. J Clin Invest, 117(10), 2740-2750. http://dx.doi.org/10.1172/JCI31809CI 2007

Koprowski, H., Steplewski, Z., Mitchell, K., Herlyn, M., Herlyn, D., \& Fuhrer, P. (1979). Colorectal carcinoma antigens detected by hybridoma antibodies. Somatic Cell Genet, 5, 957-71. http://dx.doi.org/10.1007/BF01542654

Magnai, Jr., Steplewski, Z., Koprowski, H., \& Ginburg, V. (1983). Identification of the gastrointestinal and pancreatic associated antigen detected by monoclonal antibody 19-9 in the sera of patient as mucin. Cancer Res, 43, 5489-92.

Magnani, J. L., Nilsson, B., Brockhaus, M., Zopf, D., Steplewski, Z., Koprowski, H., \& Ginsburg, V. (1982). A monoclonal antibody - defined an association with gastrointestinal cancer is a ganglioside containing sialylated lacto Nfucopentose II. J Bioi Chem, 257, 14365-9.

Pavai, S., \& Yap, S. F. (2003). The Clinical Significance of Elevated Levels of Serum CA 19.9. Med J Malaysia, 58(5), 667-72.

Rhodes, J. M., \& Ching, C. (1990). Serum diagnostic tests for pancreatic cancer. Clin Gastroenterol, 4, 835-52.

Koprowski, H., Herlyn, M., Staplewski, Z., \& Sears, H. F. (1981). Specific antigen in serum of patients with colon carcinoma. Science, 212, 53-5. http://dx.doi.org/10.1126/science.6163212

Gupta, M. K., Arciaga, R., Bocci, L., Tubbs, R., Bukowski, R., \& Deothar, S. D. (1985). Measurements of monoclonal defined antigen (CA 19-9) in the sera of patients with malignant and nonmalignant diseases: $\begin{array}{lllll}\text { comparison with carcinoembryonic antigen. } & \text { Cancer, } & 56,83 .\end{array}$ http://dx.doi.org/10.1002/1097-0142(19850715)56:2<277::AID-CNCR2820560213>3.0.CO;2-M 
Safi, F., Beger, H. G., Bittner, R., Büchler, M., \& Krautzberger, W. (1986). CA $19-9$ and pancreatic adenocarcima. Cancer, $57(4)$ $779-83$. http://dx.doi.org/10.1002/1097-0142(19860215)57:4<779::AID-CNCR2820570417>3.0.CO;2-C

Malesci, A., Tommasini, M. A., Bonato, C., Bocchia, P., Bersani, M., Zerbi, A., ... Di Carlo, V. (1987). Determination of CA 19-9 antigen in serum and pancreatic juice for differential diagnosis of pancreatic adenocarcinoma from pancreatitis. Gastrenterology, 92, 60-7.

Ohshio, G., Yamaki, K., Imamura, T., Suwa, H., Chang, C. Y., Wada, H., ... Imamura, M. (1995). Distribution of the carbohydrate antigens, DU-PAN-2 and CA19-9, in tumors of the lung. Tumori, 81, 67-73.

Mizushima, Y., Tsuji, H., Izumi, S., Hirata, H., Kin, Y., Kawasaki, A., ... Yano, S. (1991). Clinical evaluation of five tumor marker assay in patients with lung cancer. Anticancer Res, 11, 91-5.

Niklinski, J., Furman, M., Laudanski, J., \& Kozlowski, M. (1992). Prognostic value of pretreatment CEA, SCC-Ag and CA 19-9 levels in sera of patients with non-small cell lung cancer. Eur J Cancer Prev, 1(6), 401-6. http://dx.doi.org/10.1097/00008469-199210000-00002

Auberger, J., Loeffler-Ragg, J., Wurzer, W., \& Wolfgang, W. (2006). Targeted therapies in nonsmall cell lung cancer: proven concepts and unfulfilled promises. Curr Cancer Drug Targets, 6, 271-94. http://dx.doi.org/10.2174/1568009067774441780

Ghosh, I., Bhattacharjee, D., Das, A. K., Chakrabarti, G., Dasgupta, A., \& Dey, S. K. (2013). Diagnostic Role of Tumor Markers CEA, CA15-3, CA19-9 and CA125 in Lung Cancer. Indian Journal of Clinical Biochemistry, 28(1), 24-29. http://dx.doi.org/10.1007/s12291-012-0257-0

Buccheri, G. F., Ferrigno, D., Sartoris, A. M., Violante, B., Vola, F., \& Curcio, A. (1987). Tumor markers in bronchogenic carcinoma. Superiority of tissue polypeptide antigen to carcinoembryonic antigen and $\begin{array}{lllll}\text { carbohydrate antigenic determinant } 19-9 . & \text { Cancer, } & 60(1), & 42-50 .\end{array}$ http://dx.doi.org/10.1002/1097-0142(19870701)60:1<42::AID-CNCR2820600109>3.0.CO;2-3

\section{Copyrights}

Copyright for this article is retained by the author(s), with first publication rights granted to the journal.

This is an open-access article distributed under the terms and conditions of the Creative Commons Attribution license (http://creativecommons.org/licenses/by/3.0/). 